\title{
Balloon-Occluded Transcatheter Arterial Chemoembolization (b- TACE) for Hepatocellular Carcinoma Performed with Polyethylene-Glycol Epirubicin-Loaded Drug-Eluting Embolics: Safety and Preliminary Results
}

\author{
Pierleone Lucatelli $^{1}$ (D) Luca Ginnani Corradini $^{1} \cdot$ Gianluca De Rubeis $^{1}$ • \\ ${\text { Bianca } \text { Rocco }^{1} \cdot \text { Fabrizio Basilico }^{1} \text { - Alessandro Cannavale }}^{1} \cdot$ Pier Giorgio Nardis $^{1}$.

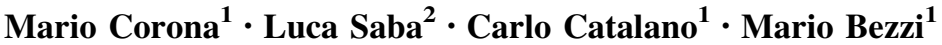 \\ Received: 28 November 2018/Accepted: 21 February 2019/Published online: 6 March 2019 \\ (C) The Author(s) 2019
}

\begin{abstract}
Purpose To report technical success, safety profile and oncological results of balloon-occluded transcatheter arterial chemoembolization using a balloon micro-catheter and epirubicin-loaded polyethylene-glycol (PEG) microsphere $(100 \pm 25 \mu \mathrm{m}$ and $200 \pm 50 \mu \mathrm{m})$ in patients with hepatocellular carcinoma (HCC).

Materials and Methods This is a single-centre, single-arm, retrospective study with 6-month follow-up. Twenty-two patients (Child-Pugh A 68\% [15/22], B in 32\% [7/22]; age $67.05 \pm 14$ years) with 29 HCC were treated in 24 procedures. Technical success is defined: ability to place the balloon micro-catheter within the required vascular segment, balloon-occluded arterial stump pressure drops and assessment of microsphere deposition. Laboratory assessment pre/post-procedural and complications were analysed,
\end{abstract}

Pierleone Lucatelli, Luca Ginnani Corradini and Gianluca De Rubeis have equally contributed to the manuscript.

Pierleone Lucatelli

pierleone.lucatelli@gmail.com

Luca Ginnani Corradini

lucaginannicorradini@gmail.com

Gianluca De Rubeis

derubeis.gianluca@gmail.com

Bianca Rocco

bianca_rocco@hotmail.com

Fabrizio Basilico

fabribasi@gmail.com

Alessandro Cannavale

alessandro.cannavale@hotmail.com

Pier Giorgio Nardis

p.nardis@gmail.com respectively, according to Common Terminology Criteria for Adverse Events (CTCAEv5) and CIRSE system. Postembolization syndrome (PES) was defined as fever and/or nausea and/or pain onset. Oncological results were evaluated using m-RECIST criteria with CT/MRI imaging at 1 and 3-6 months. In partial responder patients, pre/post-procedural tumour volume was compared.

Results Pre-planned feeder was reached in all cases. Pressure drop average was $51.1 \pm 21.6 \mathrm{mmHg}$. Exclusive target embolization was achieved in 14/24 procedures $(58.3 \%)$. Laboratory test modifications were all grade 1 . 4/24 adverse events occurred (17\%): pseudo-aneurysm of the feeder (grade 3), liver abscess (grade 2) and 2 asymptomatic segmentary biliary tree dilatations (grade 2). PES occurred in 8/24 (33\%). The complete response at 1 and 3-6 months was $44.8 \%$ (13/29) and 52.9\% (9/17), respectively. The partial response at 1 and 3-6 months was 55\% (16/29) and 4/17 (23.5\%), respectively. Among partial

\author{
Mario Corona \\ mario.corona@uniroma1.it \\ Luca Saba \\ lucasabamd@gmail.com \\ Carlo Catalano \\ carlo.catalano@uniroma1.it \\ Mario Bezzi \\ mario.bezzi@uniroma1.it \\ 1 Vascular and Interventional Radiology Unit, Department of \\ Diagnostic Service, Sapienza University of Rome, Viale \\ Regina Elena 324, 00161 Rome, Italy \\ 2 Department of Medical Imaging, Azienda Ospedaliero \\ Universitaria (A.O.U.) of Cagliari-Polo di Monserrato, \\ Cagliari, Italy
}


responder patients, the average percentage of tumour volume reduction was $64.9 \pm 27.3 \%$.

Conclusion Epirubicin-loaded PEG microsphere b-TACE is technically feasible, safe and effective procedure for HCC treatment.

Keywords Transcatheter arterial chemoembolization (TACE) - Balloon-occluded transcatheter arterial chemoembolization (b-TACE) - Drug-eluting microsphere transarterial chemoembolization (DEMTACE) - Balloon micro-catheter $\cdot$ Hepatocellular carcinoma (HCC) $\cdot$ Safety profile

\section{Introduction}

Transarterial therapies represent the standard of care for intermediate hepatocellular carcinoma (HCC) not amenable to curative treatments and an option in early stage if surgery/ablation was contraindicated [1]. Balloon-assisted TACE (b-TACE) that is a TACE procedure performed with a balloon micro-catheter inflated within the lesion's arterial feeders prior to the embolization has been recently developed in Japan by Irie et al. [2]. Balloon micro-catheter intervention works by way of blood flow modification, through a drop of intra-arterial pressure that provides a redistribution of the flow towards areas of less resistance, in this case tumour lesions [3]. Safety and efficacy of the b-TACE procedure with lipiodol in combination with anticancer drugs have been recently confirmed by some reports [4-6]. Several retrospective studies comparing oncological results on patients treated with b-TACE with lipiodol, with those of an historical matched cohort treated with c-TACE, demonstrated that b-TACE may obtain better tumour control over c-TACE in tumours up to $4 \mathrm{~cm}$ $[4,7,8]$.

Notwithstanding these advantages, it is to note that lipiodol conventional TACE (c-TACE) has shown several limitations (procedure standardization, toxicity profile, pain), overcome by the introduction of drug-eluting microsphere transarterial chemoembolization (DEMTACE) [9-11].

On the basis of those results, the combination of DEMTACE with the b-TACE may improve the oncological response in HCC. The choice of a different delivery system will provide the Interventional Oncologist with another opportunity to further address the specific needs of every single patient, in line with the modern concept of individualized medicine. To date, the combined use of these two techniques has not been reported.
The aim of this study is to describe our experience with b-TACE performed with polyethylene-glycol epirubicinloaded drug-eluting embolics in HCC patients and report about the safety and preliminary oncological results at 3and 6-month follow-ups.

\section{Materials and Methods}

This study was approved by the ethical institutional review board. Informed consent for the procedure for anonymized publication of this series of patients was obtained from all individual participants included in the study.

Between January 2018 and May 2018, we treated 22 consecutive patients (mean age $67.1 \pm 14.0$ years [range, 41-86 years]; 20 males) with 29 hepatocellular carcinoma (HCC) (average 1.3 HCC nodule/patient) [12, 13]. All patients were evaluated by a multidisciplinary board (composed of a transplant surgeon, an interventional radiologist, body radiologist and a hepatologist).

Inclusion criteria were: Child-Pugh score up to B8 and Barcelona Clinic Liver Cancer (BCLC) stage up to B and not eligible for curative treatments (surgical resection or percutaneous ablative treatments). Patients presenting with Child-Pugh $>$ B8, BCLC stage C, portal vein thrombosis (defined as the complete or partial obstruction of blood flow in the portal vein, due to the presence of a chronic, acute or neoplastic thrombus in the vasal lumen) [14], extrahepatic metastasis, and high-flow arterioportal or arteriovenous shunts, previous systemic treatment, platelet count $<50,000$, and bilirubin level $>3 \mathrm{mg} / \mathrm{dL}$, were not considered suitable for the procedure.

Diagnosis of HCC was performed according to the American Association for the Study of Liver Disease guidelines [13], using multidetector computed tomography (MDCT) $(14 / 22$ patients [64\%]) or contrast-enhanced magnetic resonance imaging (CE-MRI) (8/22 patients [36\%]).

Clinical and demographics characteristics of the cohort as well as the indications for treatment are shown in Table 1.

\section{B-TACE Technique and Outcome Measurements}

All procedures were performed through right femoral access under local anaesthetics. Liver tumour vascularization map and lesion's feeder detection (number and site) were obtained with digital subtraction angiography (anterior-posterior and right anterior oblique $25^{\circ}$ ) and dualphase cone-beam computed tomography (CBCT) performed through a 4-Fr diagnostic catheter positioned in the common hepatic artery. Based on the information obtained 
by these two imaging modalities, the best location for the balloon micro-catheter was identified, which is proximal to all lesions' feeder.
The balloon micro-catheter (Occlusafe, Terumo Europe NV, Leuven, Belgium) is 2.8 Fr micro-catheter with an occlusion balloon on the tip. The micro-balloon is made of
Table 1 Demographic characteristics

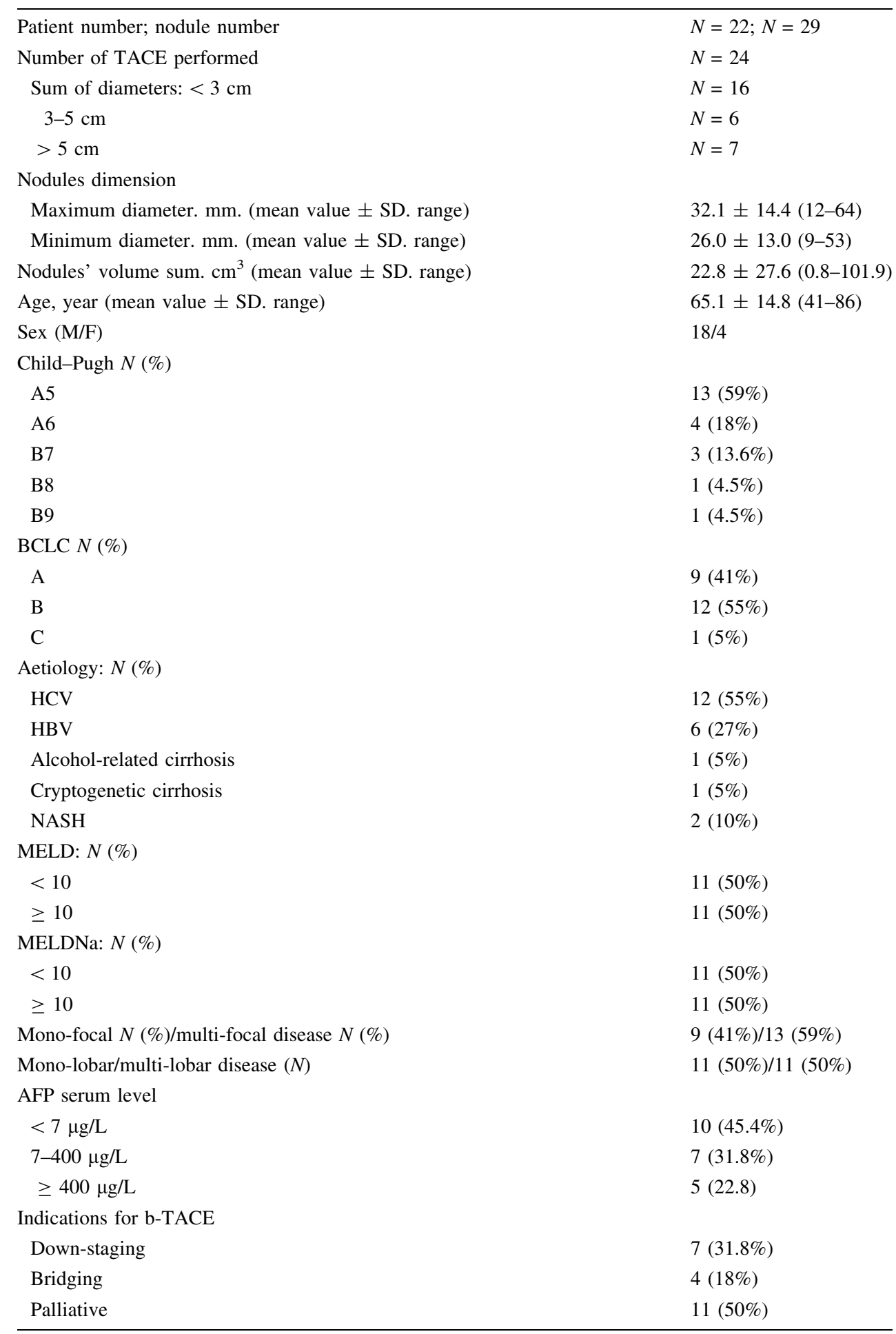

$T A C E$ Transarterial chemoembolization; $S D$ standard deviation; $M$ male; $F$ female; $H C V$ hepatitis $\mathrm{C}$ virus; $H B V$ hepatitis B virus; NASH non-alcoholic steatohepatitis; $M E L D$ model for end-stage liver disease; $A F P$ $\alpha$ - fetoprotein 
compliant polyurethane and is $10 \mathrm{~mm}$ in length. (The balloon location is indicated by two radiopaque markers.) The diameter ranges from 1 to $4 \mathrm{~mm}$, according to the volume injected. The micro-catheter can be inserted within a standard $4 \mathrm{Fr}$ angiographic catheter with a co-axial technique. The balloon micro-catheter works on a 0.014 ", platform; in this series, all procedures were performed using a hydrophilic guidewire (GT guidewire, Terumo Europe NV, Leuven, Belgium). Micro-balloon inflation was done with a solution of 1:4 of contrast media/saline.

Once the balloon micro-catheter was positioned within the target vascular segment, the arterial pressure at the tip of the micro-catheter was measured, using an invasive arterial pressure measurement kit. Thereafter, the balloon was inflated to occlude the flow and to obtain a drop of the balloon-occluded arterial stump pressure (BOASP).

The embolization was then performed, according to our routine clinical practice, with drug-eluting microsphere (Lifepearl, Terumo Europe NV, Leuven, Belgium $[100 \pm 25 \mu \mathrm{m}$ and $200 \pm 50 \mu \mathrm{m}])$ [15]), pre-loaded with $50 \mathrm{mg}$ epirubicin per syringe (2 syringes).

Embolization was performed according to the following protocol: the smaller particles $(100 \pm 25 \mu \mathrm{m}$; loaded with $50 \mathrm{mg}$ of epirubicin) were injected first. If the embolization end-points were not reached, the larger particles (200 $\pm 50 \mu \mathrm{m}$; loaded with $50 \mathrm{mg}$ of epirubicin) were then injected.

The embolization outcome measurement was composite due to the presence of the inflated balloon micro-catheter that prevented evaluation of flow stasis. Saturation of tumour vascular bed during B-TACE was indicated by the following embolization outcome measurements: reflux of microsphere despite balloon inflation following forced injection $(2 / 24$ cases [8\%]), visualization of vascular anastomosis that could determine potential non-target embolization (1/24 cases [4\%]), and manual perception of resistance to embolic injection (10/24 cases [41\%]).

The procedure was stopped once at least one of the outcome measurements was met or the maximum threshold of epirubicin (11/24 cases [45\%]), and DEM had been injected (respectively, $100 \mathrm{mg}$ epirubicin and 2 vails of DEM).

Finally, at the end of the embolization, a non-enhanced CBCT was performed, to evaluate the DEM deposition using a qualitative modification of the categorization proposed by Wang et al. [16]. The evaluation was performed as follows: complete deposition of DEM in the tumour defined as qualitative tumour contrast retention (qTCR) and qualitative defect of tumour contrast retention (qDCR). The qDCR parameter was further distinguished into qDCRa and qDCRb, by correlating the non-enhanced postprocedural CBCT with the pre-embolization arterial phase of the CBCT. When the deposition defect was correlated with the presence of a feeder that was not embolized during the procedure, this was classified as qDCRa, whereas qDCRb indicated a deposition defect not related with the presence of a non-embolized feeder but to a suboptimal DEM intra-tumoural deposition (probably due to no homogeneity of internal tumour architecture). Moreover, the presence/absence of DEM deposition within the surrounding healthy liver parenchyma was assessed and classified using a 4-point Likert scale (0: absence, 1: minimal; 2: mild; and 3: diffuse). The deposition analysis was performed by two Interventional Radiologists (BLIND and BLIND 10 and 5 years of experience) in consensus.

The b-TACE selectivity was categorized according to a dedicated score. (The score is described in detail in Table 2.)

\section{Imaging Follow-Up Timeline}

Follow-up imaging was performed at 1 and 3-6 months from b-TACE procedures with MDCT/CE-MRI and evaluated using the modified Response Evaluation Criteria in Solid Tumours (m-RECIST) [17] by an expert abdominal radiologist, not involved in the procedure.

\section{Study Outcome Measurements}

Primary outcome measurements were the technical success and the safety of the procedure.

Technical success was defined as a composite outcome measurement: ability to place the balloon micro-catheter inside the required vascular segment, drop of balloon-occluded arterial stump pressure (BOASP) and qualitative assessment of DEM deposition in the target tumour. Moreover, BOASP was correlated with the tumour volume analysis and diameter $(<3$ and $>3 \mathrm{~cm})$.

The safety of the b-TACE using DEM loaded with epirubicin was evaluated. In particular, adverse events (AEs) were recorded, and liver function tests and the routine laboratory examinations were analysed before and after the procedure (within $36 \mathrm{~h}$ ). The AEs were categorized according to the CIRSE classification system [18]. The laboratory test modifications, pre- and post-procedure,

Table 2 Embolization score

\begin{tabular}{lll}
\hline Score & Description & Procedure $(n[\%])$ \\
\hline 0 & Super-selective & $4[16.6 \%]$ \\
1 & Sub-segmental artery & $6[25 \%]$ \\
2 & Segmental artery & $8[33.3 \%]$ \\
3 & Multi-segmental & $5[20.8 \%]$ \\
4 & Lobar & $1[4.1 \%]$ \\
\hline
\end{tabular}


were evaluated according to the Common Terminology Criteria for Adverse Events (CTCAE) v5.0 [19]. Postembolic syndrome (PES) was defined as the onset of postprocedure fever and/or nausea and/or pain (pain score $>6$ on a visual analogue scale (VAS) and evaluated prior to discharge. Asymptomatic liver bile duct injuries (ALI) were defined as segmental duct dilation, biloma formation, eventually associated with jaundice [20]. Liver function tests included: serum total and conjugated bilirubin, aspartate aminotransferase (AST), alanine aminotransferase (ALT), alkaline phosphatase (ALP), gamma-glutamyl transferase (GGT) and albumin level. The routine laboratory examinations included full blood count and the coagulation profile (international normalized ratio [INR]).

The secondary outcome measurement was the oncological outcome, evaluated at 1 month and 3-6 months, according to m-RECIST criteria [17] at second line imaging (MDCT or CE-MRI). The analysis was performed on a per-patient and also on a per-procedure basis; tumour's volume modification between baseline (pre-procedure) and post-procedural (1 month and 3-6 months) was employed to stratify those patients who experienced partial response.

The volume was measured by manual segmentation of tumour margins per single slice $(1 \mathrm{~mm}$ of slice thickness for MDCT and $3 \mathrm{~mm}$ for CE-MRI). Measurements of the volumes were obtained twice in order to minimize measurement error.

\section{Statistical Analysis}

The Kolmogorov-Smirnov $Z$ test was performed to assess normality distribution for all variables tested. Continuous normal variables were expressed as mean \pm standard deviation. Continuous non-normal variables were expressed as median and confidential interval (CI) 95\%. The Student $\mathrm{T}$ test for paired sample was used for those with normality distribution, and the Wilcoxon rank-sum test for non-normal distribution. Pearson's test and partial correlation test were performed to assess the relation between BOASP and tumour volume analysis. Statistical analysis was performed, and the graph was plotted using MedCalc 8.0 software (MedCalc, Mariakerke, Belgium). $P$ values $<0.05$ were considered statistically significant, and all $P$ values were calculated using a two-tailed significance level.

\section{Results}

Twenty-four b-TACE procedures were performed in 22 patients with 29 HCC. Patients' indication for b-TACE was down-staging to liver transplantation in $7 / 22$, bridging to liver transplantation in 4/22 and tumour palliation in 11/22.
Two patients received a second b-TACE for treating a different HCC lesion fed by a different arterial feeder, whereas in 2 cases a second b-TACE (re-b-TACE) was performed for treating the residual vital tumour, either within the same catheter positioning or within another feeder. One patient received radiofrequency ablation (RFTA) of the residual viable tumour after 1-month follow-up. (Details on cohort follow-up are shown in Fig. 1). All these procedures were performed within 30 days of the CT scan performed 30 days after the first procedures.

All 22 patients fulfil the 1-month follow-up. Of 22 patients, 14 (63.6\%) performed the 3-6-month follow-up, particularly $6 / 8(75 \%)$ did not perform the second line imaging modality yet, 1/8 (12\%) follow-up not performed for worsening of clinical condition, and 1/8 (12\%) underwent to RFTA (Fig. 1).

The technical success of the procedure was $100 \%$. Planned location within the feeding artery was reached in all cases. (The selected arteries expressed as the selectivity score are summarized in Table 2).

The BOASP with the micro-balloon inflated was $64.0 \pm 15.0 \mathrm{mmHg}(\min 46 ; \max 110 \mathrm{mmHg})$, while prior to inflation it was $115.4 \pm 29.5 \mathrm{mmHg}$; therefore, the pressure drop average was $51.5 \pm 21.5 \mathrm{mmHg}$. An inverse correlation was found between BOASP drop and tumour volume reduction $(r=-0.45, P<0.05)$, and this inverse correlation remained significant considering the pre-procedural diameter of the tumour $(r=-0.45, P=0.04)$.

The subgroups (diameter $>3 \mathrm{~cm}$ or $<3 \mathrm{~cm}$ ) correlational analysis showed no differences between the two curves $(r<3 \mathrm{~cm}=-0.05$ and $r>3 \mathrm{~cm}=-0.46$; $P>0.05)$ as summarized in Fig. 2. DEM deposition showed qTCR in $14 / 24$ (58.3\%); qDCR was observed in $10 / 24(41.7 \%)$, qDCRa in $2 / 10(20.0 \%)$, and qDCRb in $8 / 10(80.0 \%)$. In $13 / 24(54.2 \%)$ of the procedures, we observed an extra-nodule DEM deposition, among these $1 / 13(7.7 \%)$ was minimal (grade 1), $8 / 13(61.5 \%)$ was mild (grade 2), and 4/13 (30.8\%) was diffuse (grade 3).

The median epirubicin dose delivered was $62.5 \mathrm{mg}$ [95\% CI 55.0-100.0], the median percentage of $100 \pm 25 \mu \mathrm{m}$ microsphere administered was $100 \%$ [95\% CI 100.0-100.0] (21/24 [88\%] completed vial of $100 \pm 25 \mu \mathrm{m})$, while the median percentage of $200 \pm 50 \mu \mathrm{m}$ infused was $22.5 \%$ [95\% CI 7.5-100.0] (8/ 24 [33\%] completed vial of $200 \pm 50 \mu \mathrm{m}$ ).

Four AEs (15\%) were reported: one pseudo-aneurysm of the inflated artery (maximum diameter $5 \mathrm{~mm}$; complication grade 3), one liver abscess (maximum diameter $42 \mathrm{~mm}$; complication grade 2) and two ALI [asymptomatic segmentary biliary tree dilatation (complication grade 2)].

The pseudo-aneurysm was treated using detachable coils (Concerto $^{\mathrm{TM}}$, Medtronic, USA), without sequelae. The 


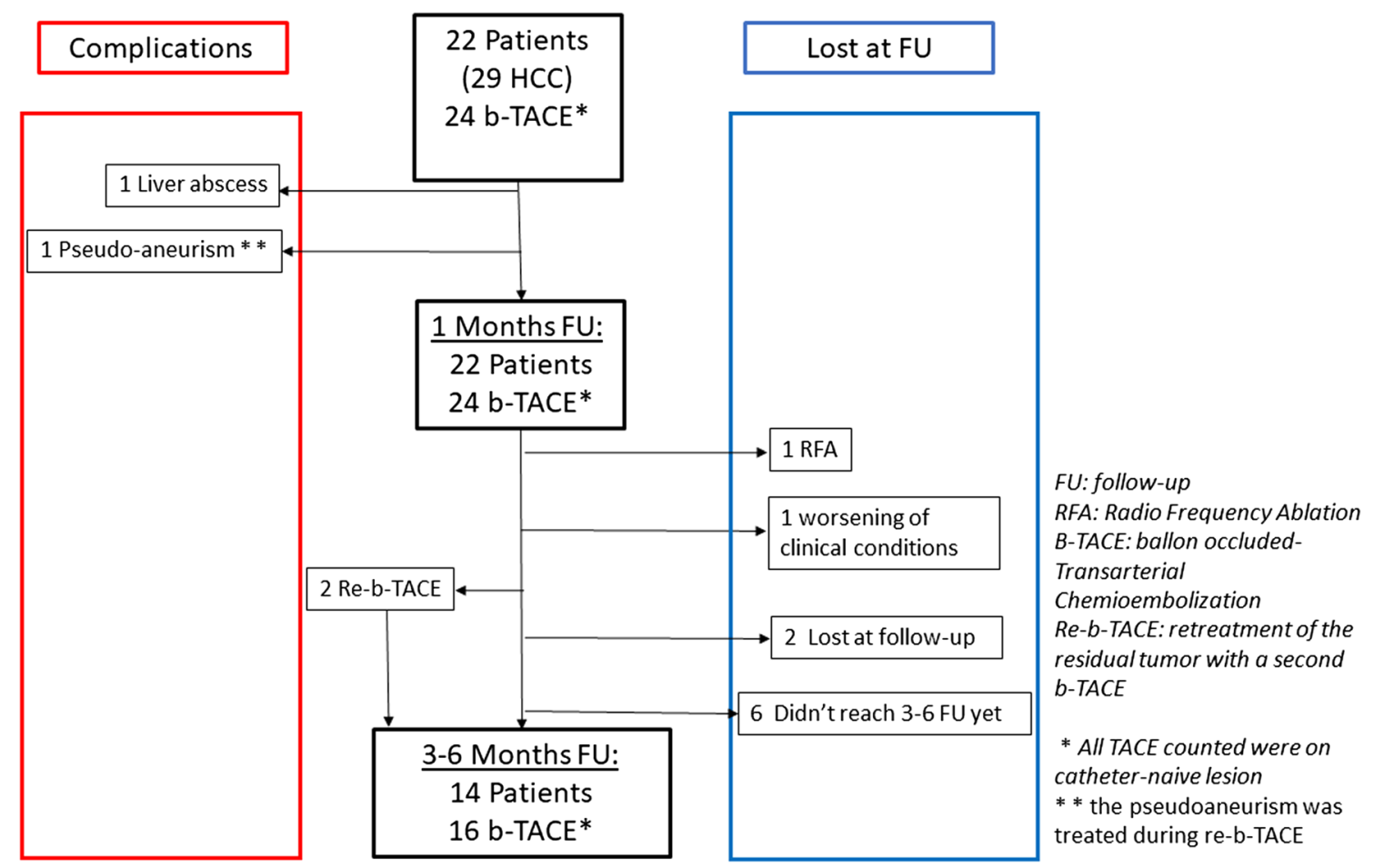

Fig. 1 Flow chart showing cohort follow-up details

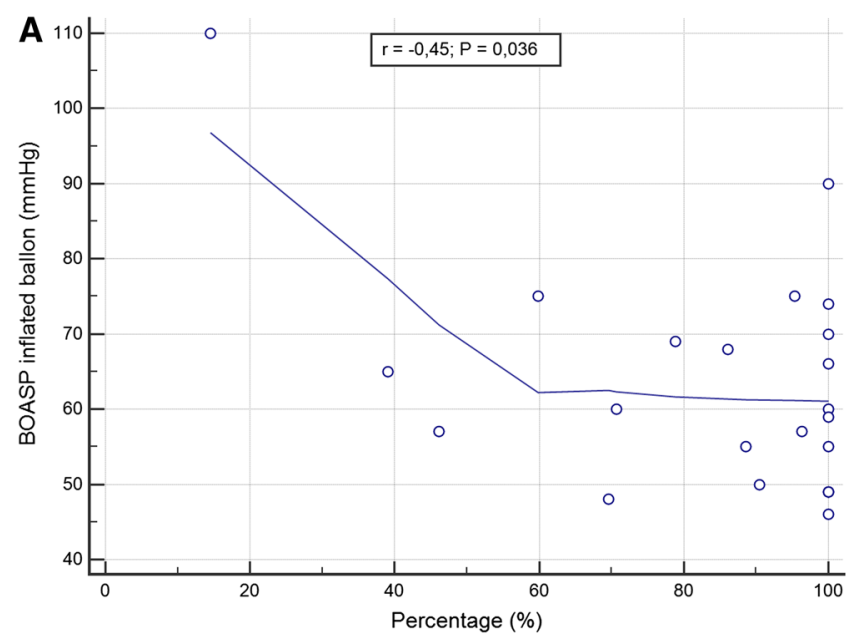

Fig. 2 Correlation analysis of balloon-occluded arterial stump pressure (BOASP) and tumour debulking. Figure A shows the inverse correlation between the BOASP and the debulking percentage; these

liver abscess was treated using antibiotic therapy only. The ALI was observed in a patient after a re-b-TACE.

After $8 / 24(33.3 \%)$ procedure, patients experienced PES. In detail, 5/24 (20.8\%) had abdominal pain (VAS score $6.7 \pm 1.2), 5 / 24(20.8 \%)$ had nausea, and $1 / 24$ $(4.2 \%)$ had low-grade fever.

Statistically significant increase of laboratory test was observed for ALT, AST, direct bilirubin, neutrophil percentage and white blood cells (for details, see Table 3). All changes were grade 1 according CTCAEv5. On the other

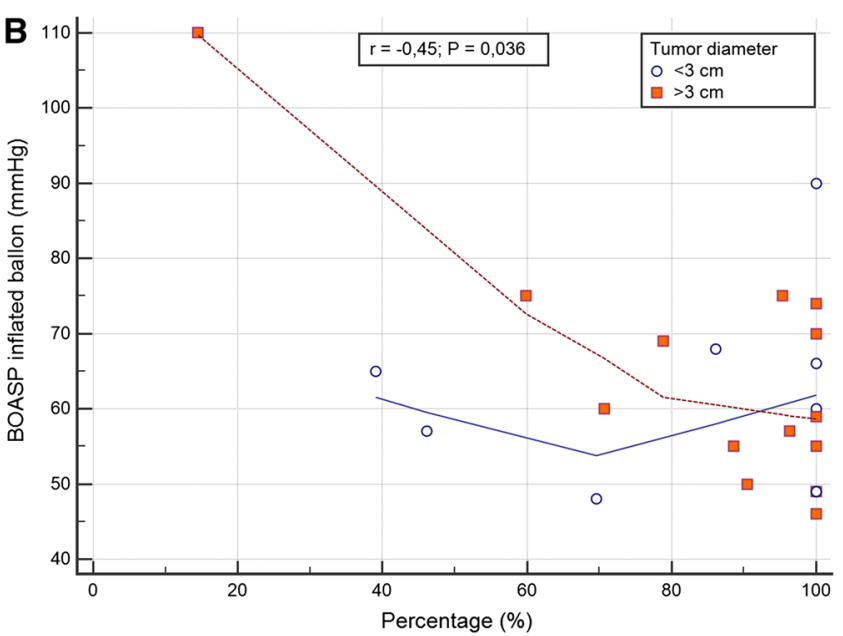

data remained statistically significant when tumour was stratified for diameter $(<3 \mathrm{~cm}$ and $>3 \mathrm{~cm})$ as highlighted in figure $\mathbf{B})$

hand, ALP and total bilirubin significantly decreased when comparing pre- and post-procedure laboratory results.

Per-patient analysis, at 1 month: in $7 / 22$ patients $(31.8 \%)$, there was a complete response (CR), and in 13/22 patients $(59.1 \%)$, there was a partial response (PR) for a total objective response (OR; [CR + PR]) of 90.9\% (20/22 patients). In two patients $(2 / 22 ; 9.1 \%)$, we observed progressive disease (PD), due to the occurrence of a new tumour in another liver segment. 
Table 3 Laboratory tests before and after balloon-occluded TACE

\begin{tabular}{|c|c|c|c|}
\hline Parameters & $\begin{array}{l}\text { Lab-pre (average } \pm \mathrm{SD} / \text { median } \\
[95 \% \mathrm{CI}])^{*}\end{array}$ & $\begin{array}{l}\text { Lab-post (average } \pm \mathrm{SD} / \text { median } \\
[95 \% \mathrm{CI}])^{*}\end{array}$ & $P$ value \\
\hline AST (IU/L) & 40.5 [22.8 to 50.4$]$ & $63.0[37.3$ to 120.1$]$ & $P=0.0003$ \\
\hline ALT (IU/L) & 24.5 [17.5 to 41.9$]$ & 57 [32.2 to 72.7$]$ & $P=0.0002$ \\
\hline ALP (IU/L) & 118 [101.6 to 143.7$]$ & 109 [96.3 to 119.4$]$ & $P=0.012$ \\
\hline Total bilirubin (mg/dL) & $0.9[0.7$ to 1.2$]$ & $0.8[0.6$ to 1.9$]$ & $P=0.03$ \\
\hline Direct bilirubin (mg/dL) & $0.4[0.3$ to 0.5$]$ & $0.4[0.3$ to 0.9$]$ & $P=0.01$ \\
\hline GGT (IU/L) & $65.5[30.7$ to 88.6$]$ & 63.0 [54.2 to 86.5$]$ & $P=0.7$ \\
\hline INR & $1.2[1.1$ to 1.4$]$ & $1.2[1.1$ to 1.3$]$ & $P=0.9$ \\
\hline Serum albumin $(\mathrm{g} / \mathrm{L})$ & $37.6 \pm 8.4$ & $36.4 \pm 6.9$ & $P=0.2$ \\
\hline $\operatorname{PLT}\left(\times 10^{3} / \mu \mathrm{L}\right)$ & $89.3 \pm 51.0$ & $82.7 \pm 46.8$ & $P=0.47$ \\
\hline WBC $\left(\times 10^{9} / \mathrm{L}\right)$ & $4.3 \pm 1.5$ & $6.2 \pm 3.3$ & $P=0.002$ \\
\hline Neutrophils $\left(\times 10^{9} / \mathrm{L}\right)$ & $1.3 \pm 3.2$ & $0.7 \pm 5.3$ & $P=0.2$ \\
\hline$\%$ Neutrophils & $56.7 \pm 11.8$ & $78.6 \pm 10.0$ & $P<0.0001$ \\
\hline
\end{tabular}

*Average $\pm \mathrm{SD}$ if the variable has a normal distribution; median $[95 \% \mathrm{CI}]$ if the variable does not have a normal distribution $A S T$ aspartate transaminase; $A L T$ alanine transaminase; ALP: alkaline phosphatase; GGT: gamma-glutamyl transferase; INR international normalized ratio; $P L T$ platelets; $W B C$ white blood cells

Per-procedure analysis, at 1 month: 10/24 (41.7\%) were on CR, and 14/24 (58.3\%) were on PR for an OR of $100 \%$ (24/24).

Among patients classified as partial responders, the average percentage of reduction in tumour volume was $64.9 \pm 27.3 \%$ with an absolute tumour reduction volume median of $6.5 \mathrm{~cm}^{3}(95 \% \mathrm{CI} 1.8$ to 13.3$)$. The baseline tumour volume was $17.7 \mathrm{~cm}^{3}$ (95\% CI 8.3 to 38.2$)$, and this value decreased to $1.2 \mathrm{~cm}^{3}(95 \%$ CI 0.0 to 8.8$)$ at 1 month $(<0.05)$ (Figs. 3, 4).

Per-patients analysis at 3-6 months showed a CR of $14.3 \%$ ( 2 out of 14 patients) and a PR $42.9 \%$ (6 out 14) leading to an OR of $57.2 \%(8 / 14)$. The PD rate was $42.8 \%$ (6 out of 14), of these, $2 / 6(33.3 \%$ ) due to a new tumour in another segment.

Per-procedure analysis, at 3-6 months: 9/17 (52.9\%) were CR, 4/17 (23.6\%) were PR, and 4/17 (23.5\%) were PD. The OR rate was $76.5 \%(13 / 17)$.

\section{Discussion}

This preliminary study shows that b-TACE performed in conjunction with epirubicin-loaded DEM is a safe procedure and is associated with a high overall tumour response.

Despite the initial complexity added to the procedure by the presence of the balloon micro-catheter and the different catheter manipulation technique, the operators were able to position and inflate the balloon micro-catheter in the selected vascular segment in all cases. This is of note, considering that $70 \%$ of balloon placements were performed in a second-order hepatic artery branch or in a more distal branch. All catheterization procedures were carried out without observing a vascular complication that could stop the embolization procedure, such as vasospasm or a dissection. In all cases, the balloon micro-catheter visibility was good enough to perform procedures.

Our data on the BOASP reduction $(63.2 \pm 14.7 \mathrm{mmHg})$ are in line with those reported by Irie et al. [2] that identified a better lipiodol dense accumulation with a BOASP lower than $64 \mathrm{mmHg}$. In our study, we observed only one case of suboptimal reduction of the BOASP (prior to inflation it was $210 \mathrm{mmHg}$; after inflation it was $110 \mathrm{mmHg}$ ). This was probably due to the vascular anatomy of this patient which had previously undergone a right hepatectomy that limited the correct placement of the balloon micro-catheter proximal to all HCC feeders. This patient, as expected, experienced a partial response to the b-TACE procedure and required a second treatment.

Regarding the site of balloon micro-catheter placement, inflation and subsequent embolization, it is worth to underline that $\mathrm{b}$-TACE was performed differently from standard DEM-TACE procedures. In fact, while DEMTACE procedures are usually performed by embolizing "as super-selective as possible within all lesion's feeders" (scores 0,1 ), b-TACE in this study was performed more proximal ("as selective as possible proximal to all lesion's feeders") by placing the balloon micro-catheter proximal to all lesions' feeders. This was done with the rationale that balloon micro-catheter inflation within the arterial segment proximal to the lesions' feeders may determine a drop of the "vis a tergo", confirmed by the drop of the BOASP, 


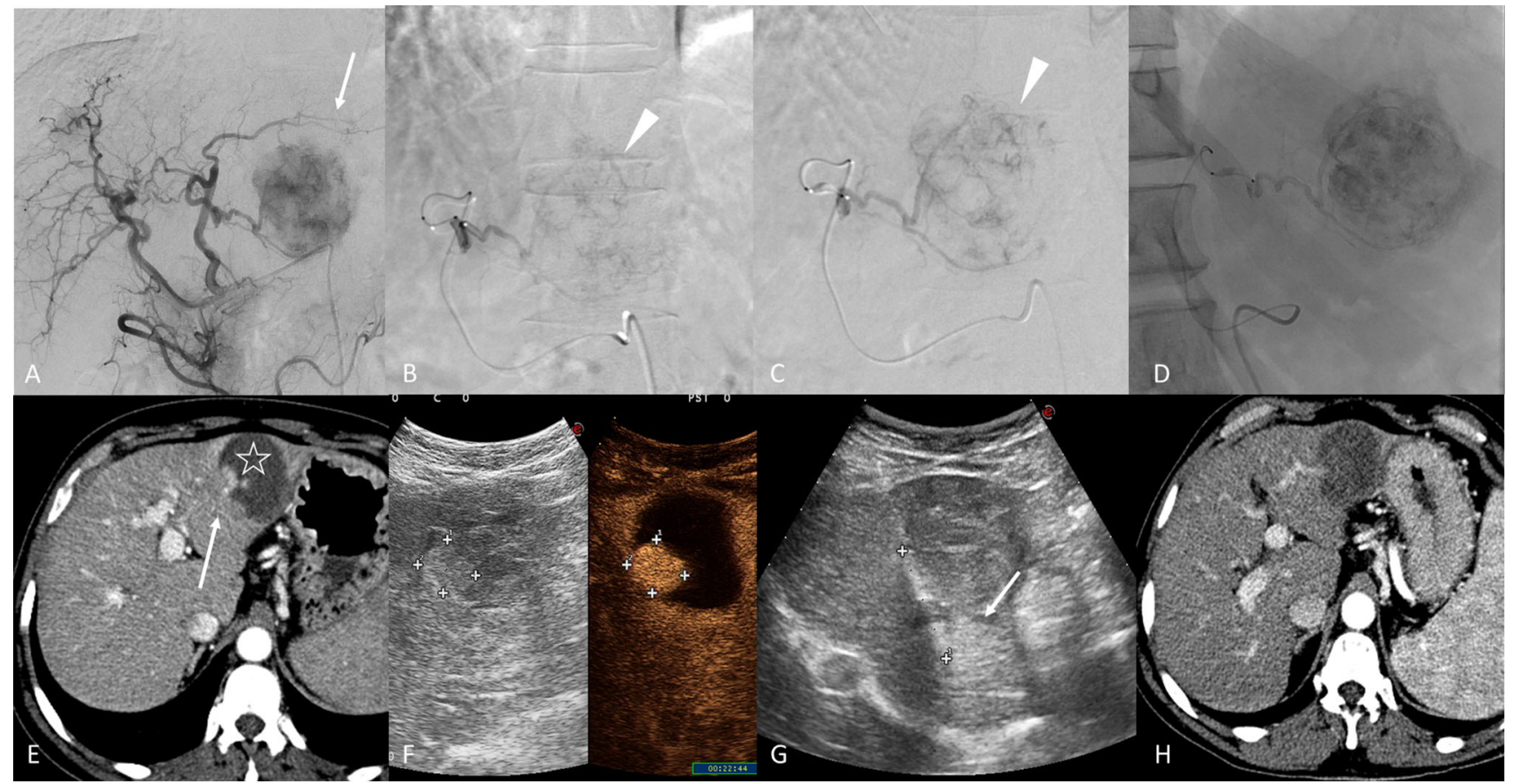

Fig. 3 A 156-year-old male with a single nodule of HCC with maximal diameter of $76 \mathrm{~mm}$ at II/III hepatic segments. Figure A, Digital subtraction angiography (DSA) performed from the common hepatic artery, shows the hypervascular structure of the HCC in the left lobe (arrow). Super-selective DSA of the tumour with deflated balloon figure B) and inflated balloon figure C) (arrowhead). Figure D shows single fluoroscopy image after the embolization.
Figure E, 1-month follow-up CT in arterial phase, highlights the partial necrosis of the nodule (star) with the presence of a hypervascular bottom (arrow) of vital residual tumour. Figure F, contrast-enhanced ultra-sound, shows the residual HCC, and figure $\mathbf{G}$ evidences the radiofrequency ablation of the lesion (arrow). Figure $\mathbf{H}$, post-procedural $\mathrm{CT}$ in arterial phase, evidences the complete response of the $\mathrm{HCC}$

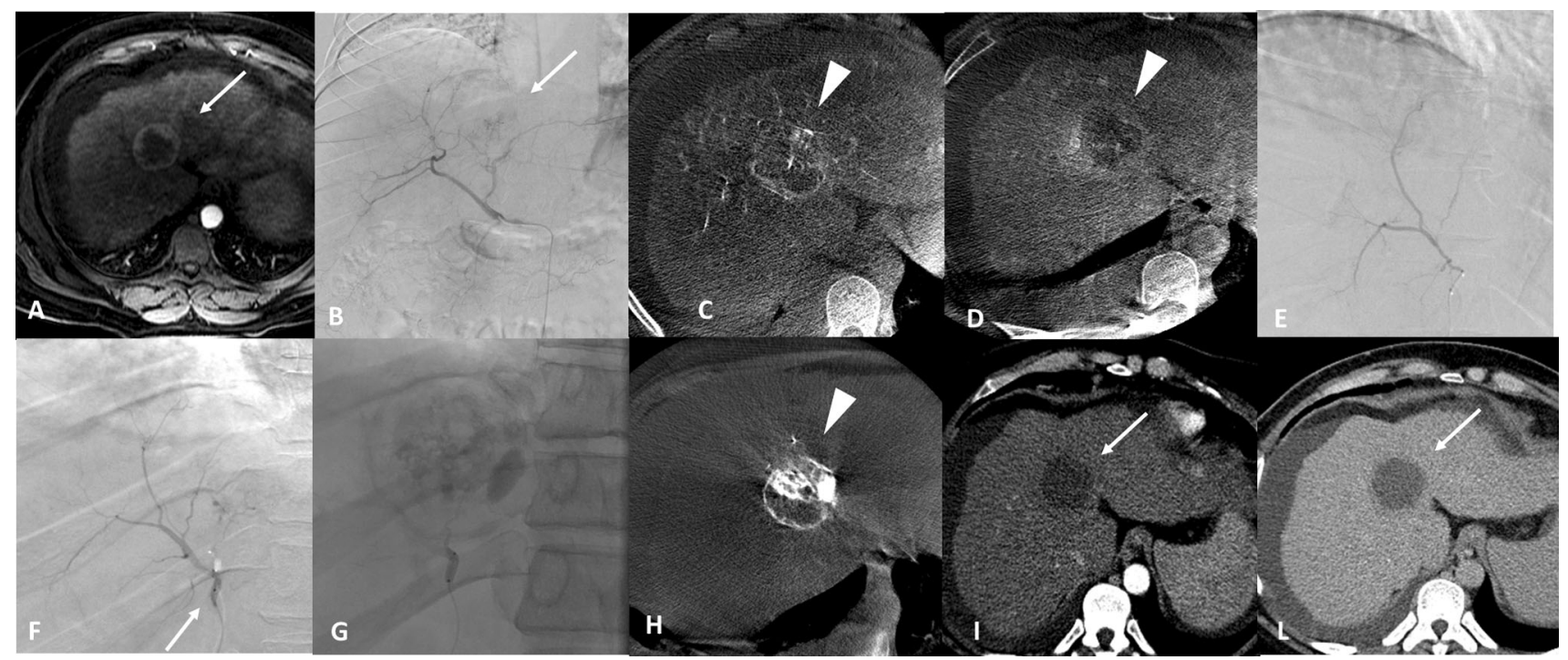

Fig. 4 A 42-year-old female with $\mathrm{HCC}$ at level of the IV hepatic segment (maximal diameter $42 \mathrm{~mm}$ ). Figure A, magnetic resonance imaging, arterial phase, shows non-homogeneous hypervascular nodule in the IV segment (arrow). The tumour is confirmed by the digital subtracted angiography (DSA) (figure B, arrow), in the conebeam CT arterial phase (figure C) (arrowhead) and cone-beam CT delayed phase (figure D) (arrowhead). Super-selective DSA of the tumour with deflated balloon figure E) and inflated balloon (arrow) figure $\mathbf{F}$ ). Figure $\mathbf{G}$ shows single fluoroscopy image after the embolization. Figure $\mathbf{H}$ non-enhanced cone-beam $\mathrm{CT}$ at the end of the procedure shows complete filling of the HCC (qTCR) (arrowhead). 1-month follow-up CT in arterial phase (figure I) and late phase (figure $\mathbf{L}$ ) demonstrates the complete response of the HCC (arrow) 
permitting flow redirection towards a low-resistance territory such as a hypervascular HCC [21]. The theoretical background underlying this procedural decision was to maximize the DEM deposition inside the tumour. This hypothesis is confirmed by the analysis performed at postprocedural non-enhanced CBCT that demonstrated presence of DEM within the target lesion. Moreover, despite that extra-nodular DEM deposition was seen in $54.1 \%$ of procedures, this was categorized as diffuse only in 4 cases and was not associated to post-procedural complications.

Of the four significant AEs, only the pseudo-aneurysm of the arterial feeder was strictly related to the use of the balloon micro-catheter. Pseudo-aneurysm formation has been reported and is correlated with the learning curve in the correct use of this device [22]. This episode occurred in fact within the first five cases of our experience. The remaining AEs represent a known complication of both the DEM-TACE procedures and b-TACE performed with lipiodol [15, 23].

Several aspects should be kept in mind when dilating the balloon micro-catheter: first, despite its compliant structure (the balloon is made of polyurethane), over-dilation may result in a vascular damage of small arteries; second, flushing the dead space of the micro-catheter balloon's line is crucial because a suboptimal flushing may impair the correct visualization of the balloon inflated to its nominal diameter; third, during micro-balloon inflation continuous BOASP monitoring is suggested because pressure drop may occur even before the required balloon diameter is reached. This third point is of particular relevance because often there is no perfect match between the recommended injected volume and the actual calibre of the inflated micro-balloon. In our opinion, inflation should be always performed under fluoroscopy as the micro-balloon needs an appropriate time (longer than a standard angioplasty balloon) to reach the required diameter.

Concerning laboratory findings, all post-procedural modifications were grade 1 according to CTCAEv5.0, despite the high degree of tumour devascularization (average debulking percentage $61.4 \pm 31.2 \%$ ) and the fact that the embolization was segmental or even more proximal in $14 / 24$ procedures $(58.3 \%)$.

With the exception of abdominal pain $(20.1 \%$ vs. $14 \%$ [23]), each aspect of PES had a lower incidence, as compared with the incidence reported in the existing literature, in particular fever was $4.0 \%$ versus $78 \%$ and $68 \%$ [23], and nausea was $20.1 \%$ versus $28 \%$ [24].

Due to the lack of data on the b-TACE performed with DEM, the comparison with the existing literature is influenced by several causes of bias: use of lipiodol, different drugs and population characteristics (e.g. number and dimension of treated tumours).
Despite these limitations, the objective response of this series is $90.9 \%$ and $58.3 \%$, respectively, at 1 and 3-6 months which are consistent with the results reported by Hatanaka et al. [6] (OR of 63.6\%), using miriplatin with lipiodol, and Kawamura et al. [25], Asayama et al. [26] and Minami et al. [23], reporting an OR of $59.6 \%, 57.1 \%$ and $56.3 \%$, respectively. This consistency is particularly relevant as our cohort of patients presented greater tumour comparing with other studies (max diameter $32.1 \mathrm{~mm} \pm$ 14.4 [range 12-64 mm] versus $2.0 \mathrm{~mm} \pm 0.9$ [23] versus $6.6 \mathrm{~mm}$ [range 9-40 mm] [26]). Further studies, with randomized control group, are necessary to deeply investigate this aspect.

Partial correlation analysis results between BOASP during balloon inflation and percentage volume reduction, categorized for tumour $<3$ and $>3 \mathrm{~cm}(r=-0.05$; $-0.46 ; P>0.05$, respectively), demonstrated a moderate inverse correlation in the subset of patients having tumours $>3 \mathrm{~cm}$ as confirmed in Fig. 2. This finding suggests a potential major benefit of the b-TACE procedure in the cohort of patients having tumour $>3 \mathrm{~cm}$.

This is of particular relevance considering the initial indication for b-TACE. Four out of five patients $(80 \%)$ with a total tumour burden outside the "up-to-seven" criteria for liver transplantation [27] were down-staged and subsequently listed in the active waiting list for liver transplantation. Among these four patients, in three cases a tumour greater than $5 \mathrm{~cm}$ was treated with a b-TACE as a standalone treatment which achieved complete necrosis, whereas the fourth patient with a tumour greater than $5 \mathrm{~cm}$, b-TACE achieved effective tumour debulking and allowed the execution of adjunctive RFA of the residual vital tissue.

Our study presents several limitations, first the small sample size sample size (22 patients), second, the nonrandomized nature of this series.

In conclusion, our series showed that the combination of b-TACE and DEM was safe and obtained a CR, at 1 and 3-6 months, of $41.7 \%(10 / 24)$ and $52.9 \%(9 / 17)$ and a PR of $58.3 \%(14 / 24)$ and $4 / 17(23.5 \%)$, respectively.

\section{Compliance with Ethical Standards}

Conflict of interest All authors declare that they have no conflict of interest.

Ethical Approval All procedures performed in studies involving human participants were in accordance with the ethical standards of the institutional and/or national research committee and with the 1964 Helsinki declaration and its later amendments or comparable ethical standards.

Informed Consent Informed consent for the procedure for anonymized publication of this series of patients was obtained from all individual participants included in the study. 
Open Access This article is distributed under the terms of the Creative Commons Attribution 4.0 International License (http:// creativecommons.org/licenses/by/4.0/), which permits unrestricted use, distribution, and reproduction in any medium, provided you give appropriate credit to the original author(s) and the source, provide a link to the Creative Commons license, and indicate if changes were made.

\section{References}

1. European Association for the Study of The L, European Organisation for $\mathrm{R}$, Treatment of C. EASL-EORTC clinical practice guidelines: management of hepatocellular carcinoma. J Hepatol. 2012;56(4):908-43.

2. Irie T, Kuramochi M, Takahashi N. Dense accumulation of lipiodol emulsion in hepatocellular carcinoma nodule during selective balloon-occluded transarterial chemoembolization: measurement of balloon-occluded arterial stump pressure. Cardiovasc Interv Radiol. 2013;36(3):706-13.

3. Sugimoto K, Saguchi T, Saito K, Imai Y, Moriyasu F. Hemodynamic changes during balloon-occluded transarterial chemoembolization (b-TACE) of hepatocellular carcinoma observed by contrast-enhanced ultrasound. J Med Ultrason. 2014;41(2):209-15.

4. Arai H, Abe T, Takayama H, et al. Safety and efficacy of balloonoccluded transcatheter arterial chemoembolization using miriplatin for hepatocellular carcinoma. Hepatol Res. 2015;45(6):663-6.

5. Maruyama M, Yoshizako T, Nakamura T, Nakamura M, Yoshida $\mathrm{R}$, Kitagaki H. Initial experience with balloon-occluded transcatheter arterial chemoembolization (B-TACE) for hepatocellular carcinoma. Cardiovasc Interv Radiol. 2016;39(3):359-66.

6. Hatanaka T, Arai H, Kakizaki S. Balloon-occluded transcatheter arterial chemoembolization for hepatocellular carcinoma. World J Hepatol. 2018;10(7):485-95.

7. Irie $\mathrm{T}$, Kuramochi M, Kamoshida $\mathrm{T}$, Takahashi N. Selective balloon-occluded transarterial chemoembolization for patients with one or two hepatocellular carcinoma nodules: retrospective comparison with conventional super-selective TACE. Hepatol Res. 2016;46(2):209-14.

8. Ogawa M, Takayasu K, Hirayama M, et al. Efficacy of a microballoon catheter in transarterial chemoembolization of hepatocellular carcinoma using miriplatin, a lipophilic anticancer drug: short-term results. Hepatol Res. 2016;46(3):E60-9.

9. Lammer J, Malagari K, Vogl T, et al. Prospective randomized study of doxorubicin-eluting-bead embolization in the treatment of hepatocellular carcinoma: results of the PRECISION V study. Cardiovasc Interv Radiol. 2010;33(1):41-52.

10. Golfieri R, Giampalma E, Renzulli M, et al. Randomised controlled trial of doxorubicin-eluting beads versus conventional chemoembolisation for hepatocellular carcinoma. $\mathrm{Br} \mathrm{J}$ Cancer. 2014;111(2):255-64.

11. Sacco R, Bargellini I, Bertini M, et al. Conventional versus doxorubicin-eluting bead transarterial chemoembolization for hepatocellular carcinoma. J Vasc Interv Radiol JVIR. 2011;22(11):1545-52.

12. Choi JY, Lee JM, Sirlin CB. CT and MR imaging diagnosis and staging of hepatocellular carcinoma: part I. Development, growth, and spread: key pathologic and imaging aspects. Radiology. 2014;272(3):635-54.
13. Bruix J, Sherman J, American Association for the Study of Liver D. Management of hepatocellular carcinoma: an update. Hepatology. 2011;53(3):1020-2.

14. Ponziani FR, Zocco MA, Campanale C, et al. Portal vein thrombosis: insight into physiopathology, diagnosis, and treatment. World J Gastroenterol. 2010;16(2):143-55.

15. Lucatelli P (2018) Polyethylene glycol (PEG)-epirubicin loaded drug-elunting embolic (DEE)-TACE procedures utilizing a combined approach with 100 - and $200-\mu \mathrm{m}$ microspheres. Iniatial Exp J Vasc Interv Radiol. https://doi.org/10.1016/j.jvir.2018.10. 026 .

16. Wang X, Erinjeri JP, Jia X, et al. Pattern of retained contrast on immediate postprocedure computed tomography (CT) after particle embolization of liver tumors predicts subsequent treatment response. Cardiovasc Interv Radiol. 2013;36(4):1030-8.

17. Lencioni R, Llovet JM. Modified RECIST (mRECIST) assessment for hepatocellular carcinoma. Semin Liver Dis. 2010;30(1):52-60.

18. Filippiadis DK, Binkert C, Pellerin O, Hoffmann RT, Krajina A, Pereira PL. Cirse quality assurance document and standards for classification of complications: the cirse classification system. Cardiovasc Interv Radiol. 2017;40(8):1141-6.

19. Services USDOHAH (2017) Common Terminology Criteria for Adverse Events (CTCAE) Version 5.0.

20. Wang Z, Wang M, Duan F, Song P, Liu F. Bile duct injury after transcatheter arterial chemoembolization: risk factors and clinical implications. Hepato-Gastroenterol. 2014;61(132):947-53.

21. Aramburu J, Anton R, Rivas A, et al. Numerical zero-dimensional hepatic artery hemodynamics model for balloon-occluded transarterial chemoembolization. Int J Numer Methods Biomed Eng. 2018;34(7):e2983.

22. Matsumoto $\mathrm{T}$, Endo J, Hashida $\mathrm{K}$, et al. Balloon-occluded transarterial chemoembolization using a 1.8-French tip coaxial microballoon catheter for hepatocellular carcinoma: technical and safety considerations. Minim Invasive Therapy Allied Technol (MITAT). 2015;24(2):94-100.

23. Minami Y, Minami T, Chishina H, et al. Balloon-occluded transcatheter arterial chemoembolization for hepatocellular carcinoma: a single-center experience. Oncology. 2015;89(Suppl 2):27-32.

24. Ishikawa $\mathrm{T}$, Abe $\mathrm{S}$, Inoue $\mathrm{R}$, et al. Predictive factor of local recurrence after balloon-occluded TACE with miriplatin (MPT) in hepatocellular carcinoma. PLoS ONE. 2014;9(7):e103009.

25. Kawamura Y, Ikeda K, Fujiyama S, et al. Usefulness and limitations of balloon-occluded transcatheter arterial chemoembolization using miriplatin for patients with four or fewer hepatocellular carcinoma nodules. Hepatol Res. 2017;47(4):338-46.

26. Asayama Y, Nishie A, Ishigami K, et al. Hemodynamic changes under balloon occlusion of hepatic artery: predictor of the shortterm therapeutic effect of balloon-occluded transcatheter arterial chemolipiodolization using miriplatin for hepatocellular carcinoma. SpringerPlus. 2016;5:157.

27. Duffy JP, Vardanian A, Benjamin E, et al. Liver transplantation criteria for hepatocellular carcinoma should be expanded: a 22-year experience with 467 patients at UCLA. Ann Surg. 2007;246(3):502-9 discussion 509-511.

Publisher's Note Springer Nature remains neutral with regard to jurisdictional claims in published maps and institutional affiliations. 